\title{
ERRATUM
}

\section{Erratum to: Comparison of Microlife BP A200 Plus and Omron M6 Blood Pressure Monitors to Detect Atrial Fibrillation in Hypertensive Patients}

\author{
Giuseppe Marazzi · Ferdinando Iellamo - Maurizio Volterrani • Mauro Lombardo • \\ Francesco Pelliccia • Daniela Righi · Fabrizia Grieco • Luca Cacciotti • \\ Luigi Iaia • Giuseppe Caminiti · Giuseppe Rosano
}

To view enhanced content go to www.advancesintherapy.com

Published online: December 13, 2014

(C) Springer Healthcare 2014

Erratum to: Adv Ther (2012) 29(1):64-70

DOI 10.1007/s12325-011-0087-0

The authors of the above-mentioned paper noticed some errors subsequent to publication and would like to make the following corrections.

On page 65 under the heading Introduction the sentence "Commercially-available

The online version of the original article can be found under doi:10.1007/s12325-011-0087-0.

G. Marazzi $(\varangle) \cdot$ F. Iellamo $\cdot$ M. Volterrani ·

G. Caminiti · G. Rosano

Department of Medical Sciences, IRCCS San Raffaele

Pisana, Roma, Italy

e-mail: Giuseppe.marazzi@sanraffaele.it

F. Iellamo $\cdot$ M. Lombardo

University of Rome, Tor Vergata, Italy

F. Pelliccia

University of Rome, La Sapienza, Italy

D. Righi

Cardian Massaia Hospital, Asti, Italy

F. Grieco

University of Naples, Federico II, Italy

L. Cacciotti

Vannini Hospital, Rome, Italy

L. Iaia

Fatebenefratelli Hospital, Isola Tiberina, Rome, Italy automated devices for self-home BP monitoring with integrated algorithms for detection of AF..." should read "Automated devices for self-home BP monitoring with integrated algorithms for detection of AF...".

On page 68 under the heading Discussion the sentence "The findings of present study on the capability of the OMRON M6 BP monitor of diagnosing $100 \%$ AF confirm those of a previous study employing an early version of Omron device (8) and extend those findings to a large population of patients with high $\mathrm{BP}^{\prime \prime}$ should read "The findings of present study on the capability of the OMRON M6 BP monitor of diagnosing $100 \%$ AF confirm those of a previous study (8) and extend those findings to a large population of patients with high BP". 